BRX-TH-521

\title{
Energy in Topologically Massive Gravity
}

\author{
S. Deser and Bayram Tekin ${ }^{12}$ \\ Department of Physics, Brandeis University, Waltham, MA 02454, USA
}

\begin{abstract}
We define conserved gravitational charges in -cosmologically extended- topologically massive gravity, exhibit them in surface integral form about their de-Sitter or flat vacua and verify their correctness in terms of two basic types of solution.
\end{abstract}

${ }^{1}$ e-mail: deser,tekin@brandeis.edu

${ }^{2}$ Address after July 15, Physics Department, Middle East Technical University, 06531 Ankara, Turkey. 
Recently [1], we defined and computed the conserved charges (particularly energy) for generic higher curvature gravity models in surface integral, flux, form about the relevant, asymptotically constant or zero curvature vacua. Our work involved the formalism of [2] about asymptotically (Anti)-de-Sitter (AdS) backgrounds in cosmological Einstein gravity. Here, we define and compute the energy in the rather different context of topologically massive gravity (TMG) [3] extended to include a cosmological term [4] [Energy was defined in the original, $\Lambda=0$, TMG [3], but not in its surface integral form in terms of generic background Killing vectors.]

We begin directly with the vacuum equations

$$
\begin{gathered}
R_{\mu \nu}-\frac{1}{2} g_{\mu \nu} R+\Lambda g_{\mu \nu}+\frac{1}{\mu} C_{\mu \nu}=0, \\
C^{\mu \nu} \equiv \frac{1}{\sqrt{-g}} \epsilon^{\mu \alpha \beta} \nabla_{\alpha}\left(R^{\nu}{ }_{\beta}-\frac{1}{4} \delta^{\nu}{ }_{\beta} R\right) .
\end{gathered}
$$

The Cotton tensor $C^{\mu \nu}$ is symmetric, traceless and identically conserved, while the parameter $\mu$ is the mass of the linearized TMG excitations at $\Lambda=0$. $C^{\mu \nu}$, being the $D=3$ conformal curvature tensor, vanishes for any Einstein space, including all external solutions of the cosmological Einstein equations, such as AdS, Schwarzschild-dS and BTZ black holes. More characteristic are geometries that obey the full TMG equations but not their Einstein part alone, some of which are given in $[5,6,7]$, but these spaces do not correspond to bounded distributions. There is as yet no known "Schwarzschild" solution, let alone more complicated asymptotically de-Sitter or flat (for $\Lambda=0$ ) ones. What has been solved explicitly is the linearized metric generated by massive spinning interiors in the $\Lambda=0$ sector [8], which will prove useful in testing our asymptotic expressions for the conserved generators.

We consider asymptotically AdS metrics ${ }^{3}$

$$
g_{\mu \nu} \equiv \bar{g}_{\mu \nu}+h_{\mu \nu}
$$

where $h_{\mu \nu}$ is a (finite) deviation about the background $\bar{g}_{\mu \nu}$ that obeys

$$
\bar{R}_{\mu \lambda \nu \beta}=\Lambda\left(\bar{g}_{\mu \nu} \bar{g}_{\lambda \beta}-\bar{g}_{\mu \beta} \bar{g}_{\lambda \nu}\right), \quad \bar{R}_{\mu \nu}=2 \Lambda \bar{g}_{\mu \nu}, \quad \bar{R}=6 \Lambda .
$$

\footnotetext{
${ }^{3}$ Our conventions are: signature $(-,+,+),\left[\nabla_{\mu}, \nabla_{\nu}\right] V_{\lambda}=R_{\mu \nu \lambda}{ }^{\sigma} V_{\sigma}, R_{\mu \nu} \equiv R_{\mu \lambda \nu}{ }^{\lambda}$.
} 
Next, we expand the field equations (1) about $\bar{g}_{\mu \nu}$; as usual, the nonlinear part is the energy momentum tensor (including matter if present):

$$
\mathcal{G}^{L}{ }_{\mu \nu}+\frac{1}{\mu} C^{L}{ }_{\mu \nu} \equiv \kappa T_{\mu \nu}
$$

where the linear cosmological Einstein and Cotton terms read respectively,

$$
\begin{aligned}
\mathcal{G}^{L}{ }_{\mu \nu} & \equiv R^{L}{ }_{\mu \nu}-\frac{1}{2} \bar{g}_{\mu \nu} R_{L}-2 \Lambda h_{\mu \nu}, \\
C_{L}{ }^{\mu \nu} & =\frac{1}{\sqrt{-\bar{g}}} \epsilon^{\mu \alpha \beta} \bar{g}_{\beta \sigma} \nabla_{\alpha}\left\{R_{L}{ }^{\sigma \nu}-2 \Lambda h^{\sigma \nu}-\frac{1}{4} \bar{g}^{\sigma \nu} R_{L}\right\} .
\end{aligned}
$$

The linear part of the Ricci tensor is

$$
R_{\mu \nu}^{L}=\frac{1}{2}\left\{-\square h_{\mu \nu}-\nabla_{\mu} \nabla_{\nu} h+\nabla^{\sigma} \nabla_{\nu} h_{\sigma \mu}+\nabla^{\sigma} \nabla_{\mu} h_{\sigma \nu}\right\}
$$

Both (6) and (7) are conserved, symmetric, and $\bar{g}^{\mu \nu} C_{\mu \nu}^{L}=0$. To every background Killing vector $\bar{\xi}^{\mu}$ corresponds a (background) conserved charge

$$
\begin{aligned}
Q^{\mu}(\bar{\xi})= & \int_{\mathcal{M}} d^{2} x \sqrt{-\bar{g}} T^{\mu \nu} \bar{\xi}_{\nu}=\int_{\mathcal{M}} d^{2} x \sqrt{-\bar{g}}\left\{\mathcal{G}_{L}{ }^{\mu \nu} \bar{\xi}_{\nu}+\frac{1}{\mu} C_{L}{ }^{\mu \nu} \bar{\xi}_{\nu}\right\} \\
& \equiv Q^{\mu} \mathrm{E}+Q^{\mu} \mathrm{C} .
\end{aligned}
$$

Next we express (9) as a 1-dimensional surface integral on the boundary. In the second paper of [1], we gave a detailed account of how this is done for the Einstein part. Here we simply quote that result and move on to the Cotton part.

$$
\begin{aligned}
Q^{\mu}(\bar{\xi})_{\mathrm{E}}=\frac{1}{8 \pi G} \int_{\partial \mathcal{M}} d S_{i} & \left\{\bar{\xi}_{\nu} \bar{\nabla}^{\mu} h^{i \nu}-\bar{\xi}_{\nu} \bar{\nabla}^{i} h^{\mu \nu}+\bar{\xi}^{\mu} \bar{\nabla}^{i} h-\bar{\xi}^{i} \bar{\nabla}^{\mu} h\right. \\
& \left.+h^{\mu \nu} \bar{\nabla}^{i} \bar{\xi}_{\nu}-h^{i \nu} \bar{\nabla}^{\mu} \bar{\xi}_{\nu}+\bar{\xi}^{i} \bar{\nabla}_{\nu} h^{\mu \nu}-\bar{\xi}^{\mu} \bar{\nabla}_{\nu} h^{i \nu}+h \bar{\nabla}^{\mu} \bar{\xi}^{i}\right\}
\end{aligned}
$$

where $i$ denotes the space direction orthogonal to the boundary $\partial \mathcal{M}$.

To write $Q^{\mu} \mathrm{C}$ in (9) as a surface ${ }^{4}$ integral, it is convenient to express $C_{L}{ }^{\mu \nu}$ in explicitly symmetric form :

$$
C_{L}^{\mu \nu}=\frac{1}{2 \sqrt{-\bar{g}}}\left\{\epsilon^{\mu \alpha}{ }_{\beta} \bar{\nabla}_{\alpha} \mathcal{G}_{L}{ }^{\nu \beta}+\epsilon^{\nu \alpha}{ }_{\beta} \bar{\nabla}_{\alpha} \mathcal{G}_{L}{ }^{\mu \beta}\right\} .
$$

\footnotetext{
${ }^{4}$ We do not go into detail about the complications of cosmological horizons for de-Sitter, as opposed to AdS. For the former, the 'boundary' we work with is inside the cosmological horizon [1].
} 
Moving the Killing vector inside the covariant derivatives, one obtains

$$
2 \bar{\xi}_{\nu} C_{L}{ }^{\mu \nu} \sqrt{-\bar{g}}=\bar{\nabla}_{\alpha}\left\{\epsilon^{\mu \alpha \beta} \mathcal{G}^{L}{ }_{\nu \beta} \bar{\xi}^{\nu}+\epsilon^{\nu \alpha}{ }_{\beta} \mathcal{G}_{L}{ }^{\mu \beta} \bar{\xi}_{\nu}+\epsilon^{\mu \nu \beta} \mathcal{G}^{L \alpha}{ }_{\beta} \bar{\xi}_{\nu}\right\}+\epsilon^{\alpha \nu}{ }_{\beta} \mathcal{G}_{L}{ }^{\mu \beta} \bar{\nabla}_{\alpha} \bar{\xi}_{\nu}
$$

The terms in the curly bracket are already in the desired surface form. To put the last term into that form, we can write it as $X_{\beta} \mathcal{G}_{L}{ }^{\mu \beta}$, where $X^{\beta}=\epsilon^{\alpha \nu \beta} \bar{\nabla}_{\alpha} \bar{\xi}_{\nu}$ is also a (background) Killing vector because $\xi$ is. This means that the last term is exactly like the Einstein part (10), but in terms of $X^{\mu}$ instead of $\bar{\xi}^{\mu}$; hence we can immediately write it in surface form as well. Now we can compactly express the conserved charges in TMG,

$$
\begin{aligned}
& Q^{\mu}(\bar{\xi})=Q_{\mathrm{E}}^{\mu}(\bar{\xi})+\frac{1}{\mu} \oint d S_{i}\left\{\epsilon^{\mu i \beta} \mathcal{G}_{\nu \beta}^{L} \bar{\xi}^{\nu}+\epsilon^{\nu i}{ }_{\beta} \mathcal{G}_{L}{ }^{\mu \beta} \bar{\xi}_{\nu}+\epsilon^{\mu \nu \beta} \mathcal{G}^{L i}{ }_{\beta} \bar{\xi}_{\nu}\right\} \\
& +\frac{1}{\mu} Q^{\mu} \mathrm{E}(\epsilon \bar{\nabla} \bar{\xi})
\end{aligned}
$$

This is the desired background-gauge invariant surface integral expression. The last term could be reduced further, but (13) is sufficiently simple.

We may now compute the energy, i.e., the charge corresponding to the time-like AdS Killing vector for the two general classes of TMG solutions. First consider Einstein spaces, for which the Einstein and Cotton parts of TMG vanish separately. Here the prototype is the SdS metric; in static coordinates it is is

$$
d s^{2}=-\left(1-r_{0}-\Lambda r^{2}\right) d t^{2}+\left(1-r_{0}-\Lambda r^{2}\right)^{-1} d r^{2}+r^{2} d \varphi^{2}
$$

In this frame,the background, namely the $r_{0}=0$ version of (14), has time-like Killing vector $\bar{\xi}^{\mu}=(-1, \mathbf{0})$. Then $X^{\mu}=(\mathbf{0}, 2 \Lambda r)$. Explicit computation shows that $Q_{E}^{0}(\epsilon \bar{\nabla} \bar{\xi})$ in (13) vanishes. It is also easy to see that the middle term does not contribute to the energy for any Einstein space since $\mathcal{G}_{L}{ }^{\mu \nu}=0$. Thus, overall, $\mathrm{SdS}$ receives no contribution to its energy from the Cotton tensor term. This was also observed in [3] for the corresponding asymptotically flat Schwarzschild solution. Finally, the first term in (13) yields the energy $E$ to be proportional $r_{0}$. 
Next, we evaluate our charges for "non-degenerate' solutions of TMG, those not already obeying the Einstein equations. Here, the linearized results of [8] suffice because in the surface expressions at infinity, only these leading parts contribute. In a suitable gauge, the metric exterior to bounded massive, spinning sources (for $\Lambda=0$ ) is given by

$$
h_{0 i}=-\epsilon_{i j} \partial_{j} W(r), \quad h_{i j}=\phi(r) \delta_{i j}, \quad h_{00}=n(r),
$$

where

$$
\begin{aligned}
& W(r)=-\frac{1}{\mu}(m+\mu \sigma)\left(\log (r)+K_{0}(\mu r)\right), \quad \phi(r)=(m+\mu \sigma) K_{0}(\mu r)+2 m \log (r), \\
& n(r)=(m+\mu \sigma) K_{0}(\mu r) .
\end{aligned}
$$

The Bessel function $K_{0}(\mu r)$ vanishes rapidly at infinity. Here, and hence also in the Einstein and Cotton tensors, the mass and spin contributions become intertwined; nevertheless, inserting this metric into our expression (13) indeed gives the desired answer. [This is rather obvious, since we are using precisely the solution whose right-hand side is a sum of localized mass $T_{00}$ and spin $T_{0 i}$ sources, and the corresponding charges are defined to be their integrals, in this weak-field system. The spin's expression has the same form as the energy's, but in terms of the rotational Killing vector.

In summary, we have constructed the conserved charges, particularly the energy and spin, in cosmological TMG for the relevant asymptotically constant curvature backgrounds. Our formula is generic, applicable to both types of solutions as seen by explicit insertion of asymptotic metric expressions. In particular, we have also demonstrated that the energy of SdS does not receive any contribution from the Cotton tensor, extending the $\Lambda=0$ result of [3]

This research was supported by NSF Grant 99-73935.

\section{References}

[1] S. Deser and B. Tekin, "Gravitational energy in quadratic curvature gravities," Phys. Rev. Lett. 89, 101101 (2002) [arXiv:hep-th/0205318]; "Energy in generic higher curvature gravity theories," Phys. Rev. D 67, 084009 (2003) [arXiv:hep-th/0212292]. 
[2] L. F. Abbott and S. Deser, "Stability Of Gravity With A Cosmological Constant," Nucl. Phys. B 195, 76 (1982).

[3] S. Deser, R. Jackiw and S. Templeton, "Three-Dimensional Massive Gauge Theories," Phys. Rev. Lett. 48 (1982) 975; "Topologically Massive Gauge Theories," Annals Phys. 140, 372 (1982) [Erratum-ibid. 185, 406.1988 APNYA,281,409 (1988 APNYA,281,409-449.2000)].

[4] S. Deser, "Cosmological Topological Supergravity" In "Quantum Theory of Gravity Ed S. M. Christensen, Adam Hilger, London (1984).

[5] R. Percacci, P. Sodano and I. Vuorio, "Topologically Massive Planar Universes With Constant Twist," Annals Phys. 176, 344 (1987).

[6] Y. Nutku, "Exact Solutions Of Topologically Massive Gravity With A Cosmological Constant," Class. Quant. Grav. 10, 2657 (1993).

[7] K. A. Moussa, G. Clement and C. Leygnac, "The black holes of topologically massive gravity," arXiv:gr-qc/0303042.

[8] S. Deser, "Gravitational Anyons," Phys. Rev. Lett. 64, 611 (1990). 